SU-HEP-4241-512

TPI-MINN-92/35-T

\title{
The Cosmological Kibble Mechanism in the Laboratory: String Formation in Liquid Crystals
}

\author{
Mark J. Bowick ${ }^{1}$, L. Chandar ${ }^{1}$ and Eric A. Schiff ${ }^{1}$ \\ Physics Department \\ Syracuse University \\ Syracuse, NY 13244-1130, USA \\ Ajit M. Srivastava ${ }^{2}$ \\ Theoretical Physics Institute \\ University of Minnesota \\ Minneapolis, MN 55455, USA
}

\begin{abstract}
We have observed the production of strings (disclination lines and loops) via the Kibble mechanism of domain (bubble) formation in the isotropic to nematic phase transition of a sample of uniaxial nematic liquid crystal. The probablity of string formation per bubble is measured to be $0.33 \pm 0.01$. This is in good agreement with the theoretical value $1 / \pi$ expected in two dimensions for the order parameter space $S^{2} / \mathbf{Z}_{2}$ of a simple uniaxial nematic liquid crystal.
\end{abstract}

July 28, 1992

1 E-mail: Bowick@suhep.bitnet; Chandar@suhep.bitnet and Schiff@suhep.bitnet.

2 E-mail: Ajit@umnacvx.bitnet ; address after 1st Sept.'92: Institute for Theoretical Physics, University of California, Santa Barbara, CA 93106. 
Nematic Liquid Crystals (NLCs) form a class of matter with a rich variety of topological defects. While the classification and static properties of these defects have been thoroughly studied [1-5] the mechanism of their formation and their dynamics is relatively unexplored in the laboratory. Recently the evolution in three dimensions of the dense tangle of string defects (disclinations) produced by a pressure-induced isotropic to nematic phase transition of a sample of NLC ("K15") was studied experimentally [6,7]. It was shown that the string length density (total length in string per volume) decays linearly in time and that the coarsening of the string density obeys the scaling hypothesis $\rho(t) \approx \frac{1}{\xi(t)^{2}}$ where the length scale $\xi(t)$ is assumed to be both the mean radius of curvature of strings and the mean separation between strings and grows in time as $t^{\nu}$ with $\nu=0.51 \pm 0.02$. It was also shown that a string loop of radius $R(t)$ shrinks in time as $R(t) \propto\left(t_{0}-t\right)^{\alpha}$, with the exponent $\alpha$ being $0.5 \pm 0.03$. This agrees with the exponent $\frac{1}{2}$ obtained from an analysis of the nematodynamic equations of a disclination line moving through a nematic medium with a constant velocity $v[1,7]$.

An important motivation for this recent work was to verify several of the string interactions postulated in the study of cosmic strings and fundamental strings. In this letter we report our observations of the formation of string defects in the supercooling of the NLC K15. Preceeding the production of strings we see the formation of uncorrelated domains (bubbles) as described theoretically by Kibble [8] some years ago in his pioneering analysis 
of the production of defects (domain walls, cosmic strings and monopoles) in the cooling of the early Universe. In our experiment disclinations are analogous to global cosmic strings rather than local cosmic strings as there are no analogues of gauge fields in simple uniaxial NLCs. The idea of testing cosmological theories of string formation in condensed matter systems was in fact proposed by Zurek [9], who suggested a cryogenic experiment to detect superfluid helium flow arising from the random formation of uncorrelated domains produced by a rapid pressure quench in an annular geometry.

NLCs are thermotropic materials typically consisting of rod-like molecules roughly $20 \AA$ in length and $5 \AA$ in width. They often have two benzene rings associated with different functions and short flexible exterior chains. They exhibit a high-temperature isotropic phase characterized by disorder with respect to translational and rotational symmetries and a low-temperature nematic phase in which there is long-range orientational order but translational disorder. The nematic phase is thus liquid with respect to translations and crystalline with respect to orientations. Configurations of NLCs are specified by a director field $\mathbf{n}(\vec{x})$. This is a three-dimensional directionless unit vector giving at each point $\vec{x}$ the local orientation of the rod molecules. An order parameter for the first-order isotropic to nematic phase transition is $\left\langle\frac{3 \cos ^{2} \theta-1}{2}\right\rangle$ where $\theta$ is the angle of the director field to some arbitrary fixed axis. This order parameter vanishes in the isotropic phase and is non-zero in the nematic phase. For $K 15$ the isotropic to nematic transition occurs at $T_{N I}=35.3^{\circ} \mathrm{C}$ at 
atmospheric pressure. The easily accessible temperatures (typically $0^{\circ}-200^{\circ} \mathrm{C}$ ) of nematic phase transitions is one reason these systems are simple to work with in the laboratory (especially compared to superfluid helium).

The effective energy density describing NLCs is similar to that of an $O(3)$ non-linear sigma model except that the NLC system is invariant only under simultaneous spatial and internal rotations rather than independent spatial and internal rotations [6]. This means that the effective energy density for NLCs consists of three terms of different symmetry associated with splay, twist and bend deformations respectively [5], as well as a surface term. Corresponding to each term is a potentially different elastic (Frank) constant. The non-linear sigma model field theory is recovered only in the isotropic limit in which all three elastic constants are equal (the one-constant approximation).

NLCs exhibit three classes of topological defects. The space of ground-state nematic configurations $\mathcal{M}$ (ground-state manifold) is given by $S O(3) / D_{\infty}$, where $S O(3)$ is the symmetry group of the disordered (isotropic) phase and $D_{\infty}$ is the unbroken subgroup consisting of rotations about the molecular axis and $180^{\circ}$ rotations about axes perpendicular to the molecular axis. This space is isomorphic to $S^{2} / \mathbf{Z}_{2}$, the two-sphere with antipodal points identified, which in turn is isomorphic to the projective plane $\mathbf{R P}^{2}$. The existence of topological defects is determined by the homotopy groups of the ground-state manifold. The non-vanishing homotopy groups of $\mathcal{M}$ are $\pi_{1}(\mathcal{M})=\mathbf{Z}_{2}, \pi_{2}(\mathcal{M})=\mathbf{Z}$ and $\pi_{3}(\mathcal{M})=\mathbf{Z}$. 
Thus there are $\mathbf{Z}_{2}$ disclinations (string or line defects), integrally-charged monopoles (point defects) and integrally charged texture defects (whose field theory analogues are Skyrmeons) associated with non-trivial elements of the first, second and third homotopy groups respectively [2]. In this letter we will be concerned only with the $\pi_{1}$ defects. Note that non-trivial strings arise from the lack of polarity of liquid-crystal molecules which gives rise to the director field identification $\mathbf{n} \equiv-\mathbf{n}$. This is encoded in the $\mathbf{Z}_{2}$ isotropy group of $\mathcal{M}=S^{2} / \mathbf{Z}_{2}$. A path in $\mathcal{M}$ which winds from $\mathbf{n}$ to $-\mathbf{n}$ as one goes around a closed loop in space will not be continuously deformable to a point and hence will represent a non-trivial disclination (string) defect.

To see more clearly the director field configurations associated with disclinations we now give a very brief description of them. The basic string defect along the $z$-axis is characterized by the planar configuration of the director field [5]

$$
n_{x}=\cos \phi, \quad n_{y}=\sin \phi \quad \text { and } \quad n_{z}=0,
$$

where we have taken the director to lie in the $x-y$ plane. For an isotropic NLC (all elastic constants equal) the equations of motion minimizing the free energy are

$$
\nabla^{2} \phi=0
$$

Solutions independent of the radial coordinate $r=\sqrt{x^{2}+y^{2}}$ are given by

$$
\phi=s \alpha+c,
$$


where $\alpha=\tan ^{-1}(y / x)$ and $c$ is a constant between 0 and $\pi$. In the core of the string (of typical radius $100 \AA$ ) the director changes discontinuously and the most reasonable assumption is that the system is in the isotropic phase [4]. The parameter $s$ labels the strength of the disclination. As the core of the string is encircled the director rotates by an angle $2 \pi s$. The basic disclinations are the type- $\pm \frac{1}{2}$ disclinations. They can be continuously rotated into each other and so are topologically equivalent. Integral strength disclinations are continuously deformable to trivial configurations without a singular core by escape into the third dimension [2] and hence are topologically trivial although their dynamics is still of considerable interest. The energy density per unit length of a disclination is proportional to the square of its strength and hence the strength $\pm \frac{1}{2}$ disclinations dominate the statistical mechanics of these systems.

The formation of topological defects in a phase transition as described by Kibble [8] in the context of the early Universe, arises due to the existence of uncorrelated regions of space (domains). As these domains come into contact with each other, the variation of the order parameter field from one domain to another may be such that topological defects get trapped at the junctions of these domains. The probability of defect formation can be calculated by assuming that the order parameter field varies randomly from one domain to another. Let us illustrate this by considering a simpler case of 2 spatial dimensions when the ground state manifold is a circle $S^{1}$. The value of the order parameter field in 
the ground state is then given by an angle $\theta$ between 0 and $2 \pi$. The strings will arise when $\theta$ winds by $2 \pi$ around a closed path in space. Consider now the situation when three domains meet at a point and further that $\theta$ takes random values (say $\theta_{1}, \theta_{2}$ and $\theta_{3}$ ) in these three domains. The value of $\theta$ in between any two domains will be governed by energetic considerations and will be such that it amounts to smallest variation of $\theta$ from one domain to another. With this, the string formation at the junction will correspond to the case when one of the angles (say $\theta_{3}$ ) is such that $\theta_{1}+\pi<\theta_{3}<\theta_{2}+\pi$. [Assuming that $\theta_{2}+\pi>\theta_{1}+\pi$, otherwise the order of $\theta_{1}$ and $\theta_{2}$ should be reversed. All angles here are taken $\bmod (2 \pi)$.] The maximum and minimum values of this angular span available for $\theta_{3}$ for string formation are $\pi$ and 0 with an average of $\pi / 2$. As $\theta_{3}$ can assume any value between 0 and $2 \pi$, the probability that a string will form at the junction is $1 / 4$. The calculation of probability of formation of other defects (and for other ground state manifolds) can be carried out along similar lines. For NLCs the ground state manifold is $\mathbf{R P}^{\mathbf{2}}$ and the probability of string formation in 2 spatial dimensions can be calculated to be $1 / \pi($ see $[10])$.

It is known that for NLCs, the isotropic-nematic phase transition is of first order [11] and proceeds through the proccess of bubble nucleation. As a sample of NLC supercools below $T_{N I}$ it becomes favorable for domains (bubbles) of true ground state (nematic phase) to form in the false ground state medium (isotropic phase). Very small bubbles have 
too much surface energy and collapse again. Bubbles beyond a certain size (the critical bubbles) gain enough bulk energy to overcome the surface energy and begin to grow (see [12] for a discussion of vortex formation by bubbles). The director field will randomly take values in $\mathcal{M}$ in different bubbles and will be roughly uniform inside a given bubble (for energetic reasons). As the bubbles collide, the actual correlation length will depend on the bubble size as well as on the rate of bubble collision and when bubble collisions are frequent, each bubble will represent an uncorrelated domain as used in the Kibble mechanism. [The correlation length here is similar to the horizon in the cosmological setting in which different domains are causally disconnected and hence cannot communicate with each other.] As the system cools the initially chaotic variation of the director field will smoothen as various neighboring domains coalesce and attempt to lower gradient energies by aligning their associated director fields. As described above, however, there may be topological obstructions to neighboring domains aligning completely. If there are nontrivial paths in $\mathcal{M}$ traced out by neighboring domains then $\pi_{1}$ ( string or disclination) defects are trapped in the system somewhat like flux tubes in Type-II superconductors. This is the process we have observed in the experiment described below. One of the key quantities of interest in this mechanism of string production via bubbles is the probability of string formation per bubble. We have determined the typical domain (bubble) size and net string length produced thereby experimentally determining the value of the string 
formation probability.

For our observations we used the NLC $K 15$ (4-cyano-4'-n-pentylbiphenyl) manufactured by BDH Chemicals, Ltd.. We employed an Olympus, Inc. model BH phase contrast microscope with a 10X objective. The microscope was equipped with a monochrome television camera and a standard videocassette recorder.

We tried two different approaches to observing the isotropic - nematic phase transition. In the first a freely suspended film of $K 15$ was created by dipping a wire loop $3 \mathrm{~mm}$ in diameter in a sample of $K 15$. This wire loop was soldered to an $18 \Omega$ resistor which acted as a heater. The loop and heater assembly were fastened to an aluminum slide with a keyhole machined to accomodate them. With this apparatus we first heated the wire loop until the film entered the isotropic phase; in this phase the microscope image was completely structureless. We then switched off the electrical heater and allowed the film to cool. A similar approach was used previously by Chuang, et al [6] for the observation of the evolution of string defects, which formed very rapidly as the film entered its nematic phase. Our results in this regime largely reproduced this previous work.

This apparatus proved to be unsuitable for observing homogeneous bubble formation and the subsequent generation of strings. A circular front of nematic phase formed at the boundary of the film and propagated through the central region of the film before we could finish observing the evolution of the homogeneously nucleated bubbles. We tried to reduce 
this effect by slowing down the cooling rate by slowly reducing the current through the resistor and by shining an auxiliary illuminator during the measurements. This improved the production of bubbles but did not eliminate the problem of the circular front. We therefore tried observing the phase transitions by using a different method by using a thin drop of K15 placed on an untreated, clean microscope slide. For this arrangement heating was done using an illuminator; we were able to obtain satisfactory images of bubble formation and evolution.

One set of such images is reproduced in Fig. 1. Fig. 1(a) shows the numerous small isolated bubbles of nematic phase which form first. Fig. 1(b) and 1(c) show the images at short intervals later, as the nematic bubbles increase in size. This occurs both by natural growth and also by coalescence. In the next stage the organization of the NLC into bubbles is replaced by an image of a homogeneous medium with entangled strings (Fig. 1(d)), which further evolve by straightening, shrinking, and excising small loops of closed string (Fig. 1(e)). String dynamics have been very well described previously $[6,7]$. The film becomes increasingly homogeneous in appearance on longer time scales, as the string pattern coarsens.

We analyzed series of images such as Fig. 1 to estimate the string to bubble ratio. In counting the number of bubbles we have to decide which bubbles are relevant for string formation. For example between Fig. 1(b) and 1(c) small bubbles seem to just get absorbed 
by bigger bubbles and we do not see them contributing to any string formation. This observation is consistent with the results of the numerical simulation in [12] where the collision of two large (critical) bubbles with one small (subcritical) bubble was considered and it was found that even if a string forms in such a collision, it gets pushed out of the smaller bubble due to large momentum of the fast expanding walls of the large bubbles. Even in the collision where all the bubbles involved are very small, we have not observed any string formation. This could again be because a string will get trapped at the collision point only when the bubble walls have sufficient momentum which may not happen if bubbles are very small. Even if some strings do form in the collisions of very tiny bubbles, these strings will be very small (for isolated collisions) and will dissappear quickly by shrinking. As we count the strings at a little later stage anyway (when we can clearly distinguish them), these small strings would have dissappeared by that time and hence no error will be caused by the neglect of very small bubbles.

Guided by these considerations, we made a judgement of a typical size for the bubble which seems to be large enough to contribute to string formation and then consider coalescence and growth of such bubbles. Bubbles $r$ times the average bubble size are assumed to have arisen from the coalescence of $r$ average bubbles (as can be verified by looking at the earlier stages of the bubble formation) and are thus counted $r$ times.

The next step is to look at a photograph showing these $N$ bubbles which will eventually 
expand to fill up the entire region of the photograph (of area $A$ ). As the bubbles will coalesce they will not remain spherical but will actually squeeze into gaps. The final image will look roughly like a rectangular region filled by $N$ squares (with typical side of a square $d$ roughly equal to $\sqrt{A / N}$ ). We estimated a count of about 55 "effective" bubbles in Fig. 1(c); the typical length scale was about $80 \mu \mathrm{m}$. Our estimate of the probability that each of these bubbles will lead to a string upon final coalescence was made as follows. Imagine that the rectangular image above is a lattice of area $A$ with $m$ rows and $n$ columns such that $N=m n$. As strings form because of mismatches of the director at bubble interfaces the maximum possible string length $L_{\max }$ is given by the total sum of the lengths of all the links on the lattice which is $2(N A)^{1 / 2}$. For Fig. $1(\mathrm{c})$ we estimate $L_{\max }=8.7 \mathrm{~mm}$. We then examine a photograph of the earliest possible stage when one can discern strings clearly and measure the total length of string $L_{s}$; in Fig. 1(d) we obtained $2.7 \mathrm{~mm}$. The probability of string formation is then taken to be $P=L_{s} / L_{\max }=0.31$.

We repeated this analysis on several sequences, obtaining $P=0.33 \pm 0.01$ for the string formed per bubble. Systematic errors are not negligible; we believe that this measurement provides a lower bound on the true value of $P$ for two reasons. First the total string length will have decreased from its initial value by the time we are able to clearly resolve and measure $L_{s}$. Secondly strings which wander significantly in the direction normal to the plane of focus (but are still formed due to top bubble layers which we observe) will have 
their length underestimated. The actual underestimation may not be too bad since string wiggling will be dampened in the time between their formation and our measurement of their length. Further there is a selection effect in the experiment since we are able to see strings clearly only when they do not deviate significantly from the plane of focus. Thus we are effectively counting planar strings. This will suppress the effects of the threedimensionality of the bubble layers. In other words, the bubbles we see are only the top few layers; similarly we see the strings at the time they are roughly confined to these top layers. The numbers we obtain, therefore, correspond to an effectively two-dimensional situation. Note that for strictly 2 dimensional situation, the strings will form perpendicular to the plane formed by the bubble layer. In the present case, however, strings will bend around in the plane as they come towards the top (where the director will eventually become normal to the surface this being the preferred boundary condition for NLC-air interface) as well as when they squeeze through the bubble layers underneath them.

We note here that the string to bubble ratio we have measured is in good agreement with the value $1 / \pi \approx 0.32$ obtained by Vachaspati [10] for the ground-state manifold $S^{2} / \mathbf{Z}_{2}$ in two dimensions, especially considering the above arguments that our estimates for $P$ correspond to an effectively 2 dimensional situation.

We now turn to another interesting phenomenon we are able to observe in this experiment. We first cool our sample through the nematic phase transition so that disclinations 
are produced and then superheat from the nematic phase to the isotropic phase. Spherical isotropic bubbles are produced in the nematic medium. These have a somewhat different more transparent appearance than the nematic bubbles in an isotropic medium because of the different optical properties of the two phases. In this way it is quite easy to distinguish the different types of bubble. We observe very clearly in this process the heterogeneous nucleation of isotropic bubbles along disclination lines like beads on a necklace, see Fig. 2. This nucleation process is of great technological and theoretical interest $[13,14]$. We feel that NLCs are a good testing ground to check experimentally many of the theoretical predictions concerning the mechanism of the nucleation process such as the size distribution of bubbles.

Finally we point out that the implications of the work described here extend beyond the dynamics of the formation of disclinations in nematic liquid cystals because of the close analogies with the formation of other one-dimensional topological defects such as screw dislocations in crystals and vortices in superfluid helium $[5,9]$.

We thank Bernard Yurke for his valuable advice during his visit to Syracuse University. We would also like to express very warm thanks to Edward Lipson and his biophysics group at Syracuse University for their generosity, both in sharing their microscope apparatus and in instructing us in its use. Without their support the research reported here would not have been possible. AMS would like to thank Mark Hindmarsh for useful dis- 
cussions on theoretical estimates of string formation probability. The research of MJB was supported by the Outstanding Junior Investigator Grant DOE DE-FG02-85ER40231. The research of LC was supported by a Syracuse University Fellowship. The research of AMS was supported by the Theoretical Physics Institute at the University of Minnesota and by the US Department of Energy under contract number DE-AC02-83ER40105. MJB acknowledges the hospitality of the theoretical physics group at the Università di Roma, "Tor Vergata" where this manuscript was completed. 


\section{References}

[1] P.G. de Gennes, The Physics of Liquid Crystals (Clarendon Press, Oxford, 1974).

[2] N.D. Mermin, Rev. Mod. Phys. 51 (1979) 591.

[3] Y. Bouligand, in Les Houches Session XXXV, Physics of Defects, R. Balian, Ed. (North Holland, Dordrecht, 1981).

[4] M. Kléman, Points, Lines and Walls in Liquid Crystals, Magnetic Systems and Various Ordered Media (Wiley, Chichester, 1983).

[5] S. Chandrasekhar and G.S. Ranganath, The Structure and Energetics of Defects in Liquid Crystals, Adv. Phys. 35 (1986) 507-596.

[6] I. Chuang, R. Durrer, N. Turok and B. Yurke, Science 251 (1991) 1336; B. Yurke, A.N. Pargellis, I. Chuang and N. Turok, Princeton University preprint PUPT-91-1274 (1991).

[7] I. Chuang, N. Turok and B. Yurke, Phys. Rev. Lett. 66 (1991) 2472.

[8] T. Kibble, J. Phys. A9 (1976) 1387.

[9] W.H. Zurek, Nature 317 (1985) 505.

[10] T. Vachaspati, Phys. Rev. D44 (1991) 3723.

[11] L. Onsager, Ann. N.Y. Acad. Sci. Vol. 51 (1949) 627.

[12] A.M. Srivastava, Phys. Rev. D45 (1992) R3304; Phys. Rev. D46 (1992), in press.

[13] J.D. Gunton, M.San Miguel and P.S. Sahni, in Phase Transitions and Critical Phe- 
nomenon, Eds. C. Domb and J.L. Lebowitz (Academic Press, New York, 1983), Vol.8, pp. $269-482$.

[14] L. Monette and W. Klein, Phys. Rev. Lett. 68 (1992) 2336. 


\section{Figure Captions}

Fig. 1. A series of five images showing the isotropic to nematic phase transition in a drop of K15 on an untreated microscope slide; the images show a width of $0.7 \mathrm{~mm}$ of the drop. Note the stages of bubble nucleation and growth, bubble coalescence and string formation, and string coarsening. The delay times for each image (referred to the first frame showing discernible bubbles) are (a) $2 \mathrm{~s}$, (b) $3 \mathrm{~s}$, (c) 5 $\mathrm{s}$, (d) $11 \mathrm{~s}$, and (e) $23 \mathrm{~s}$. Scale of the pictures is given in (f).

Fig. 2. This series of three pictures shows the heterogeneous nucleation of isotropic bubbles along disclination lines. The delay times for each image, referred to the picture in (a), are (b) $2 \mathrm{~s}$, and (c) $4 \mathrm{~s}$. Scale is given in (d). 